\title{
ANÁLISIS DE CONECTIVIDAD Y DENSIDAD DE LA RED VIAL EN LA RESERVA NATURAL DEL IBERÁ CON SISTEMAS DE INFORMACIÓN GEOGRÁFICA (SIG)
}

\author{
Prof. Federico Carlos Arias \\ Becario Doctoral \\ Consejo Nacional de Investigaciones Científicas y Técnicas (CONICET). \\ ariasfedericocarlos@conicet.gov.ar \\ Dr. Osvaldo Daniel Cardozo \\ Prof. Adjunto. Cátedra de Sensores Remotos \\ Departamento de Geografía. Facultad de Humanidades. Universidad Nacional del Nordeste (UNNE). \\ odcardozo@hum.unne.edu.ar \\ Mgter. Miguel Alejandro Parras \\ Auxiliar Docente de 1ra. Cátedra de Técnicas en Geografía I \\ Departamento de Geografía. Facultad de Humanidades. Universidad Nacional del Nordeste (UNNE). \\ aleparras@hotmail.com
}

\section{RESUMEN}

Desde el punto de vista del sistema de transporte la red es un elemento fundamental a la hora de explicar cómo funciona un determinado territorio. En este sentido, la Geografía ha incorporado de manera efectiva diferentes técnicas cuantitativas y los Sistemas de Información Geográfica, para analizar los desequilibrios espaciales.

El área de la Reserva Natural de los Esteros del Iberá tiene escasa información referida a sus características humanas, y en lo particular referido a la infraestructura vial. Por esa razón, este trabajo pretender ser un aporte al conocimiento de las características estructurales de la red de transporte interna en la Reserva [1].

Es por ello que, se pretende conocer el grado de conectividad de la red vial en la Reserva mediante el cálculo de índices propios de la Teoría de Grafos, además de identificar patrones espaciales en la distribución de la infraestructura vial.

La secuencia metodológica sobre la cual se organizó el trabajo comprendió la: a) recopilación de información cartográfica, estadística y documental, b) consulta a fuentes claves de la temática como el Instituto Geográfico Nacional, la Dirección Provincial de Vialidad de Corrientes, etc. c) relevamiento e inventario de la red de transporte por digitalización c) cálculo de variables como longitud de red interior, densidad Kernel, e índices Alfa y Gamma, d) elaboración de cartografía y cuadros estadísticos, g) interpretación de la información resultante.

Los resultados indican que el área de la Reserva presenta una red vial con baja densidad y conectividad producto de una distribución heterogénea sobre el espacio. Esta cuestión se mantiene en las dos fuentes de información analizadas, aunque presentan diferentes participaciones en los departamentos.

\section{PALABRAS CLAVES}

Esteros del Iberá; Corrientes; análisis de redes; índices Gamma y Alfa; Densidad focal.

Publicado en formato digital: Prof. Federico Carlos Arias, Dr. Osvaldo Daniel Cardozo y Mgter. Miguel Alejandro Parras. ANÁLISIS DE CONECTIVIDAD Y DENSIDAD DE LA RED VIAL EN LA RESERVA NATURAL DEL IBERÁ CON SISTEMAS DE INFORMACIÓN GEOGRÁFICA (SIG). Revista Geográfica Digital. IGUNNE. Facultad de Humanidades. UNNE. Año 13. № 26. Julio - Diciembre 2016. ISSN 1668-5180 Resistencia, Chaco. 
[1] Distintos avances y resultados parciales del trabajo fueron presentados por los autores en distintos congresos: en $\mathrm{XV}^{\circ}$ Encuentro de Profesores en Geografía del Nordeste. Universidad Nacional del Nordeste. 2014. y en: III Jornada Provincial de Infraestructuras de Datos Espaciales y la Vo Reunión de Usuarios de la Tecnologías de la Información Geográfica del NEA. UNNE-CONICET. DIT-Ministerio de Planificación y Ambiente. 2015.

\title{
ROAD NETWORK CONNECTIVITY AND DENSITY ANALYSIS WITH GEOGRAPHIC INFORMATION SYSTEMS (GIS) IN THE NATURAL RESERVE OF IBERA
}

\begin{abstract}
(*)
In the field of transportation system the road network is a main component explaining to how a given territory works. In this sense, the Geography has effectively incorporated quantitative and GIS techniques to analyze spatial imbalances.

The natural reserve of lberá wetlands has little information regarding its human characteristics and especially about the road infrastructure. This paper intends to contribute some knowledge to the structural characteristics of the transport network in the Reserve.

The paper intends to show the connectivity degree of the road network in the Reserve through the calculation of indexes as part of Graph Theory, in addition to identifying spatial patterns in the distribution of road infrastructure.

The work was organized included a compilation of cartographic, statistical and documentary information; consultation of key sources of the subject such as the National Geographic Institute, the Road Direction of Corrientes, etc. Also a survey and inventory of the transport network by digitization, calculation of variables such as internal road network length, Kernel density, Alpha and Gamma indexes, mapping, statistical tables and the interpretation of resulted information.

The results indicate that the Reserve area presents a road network with low density and low connectivity due to a heterogeneous distribution over space. This situation remains in the two sources of information analyzed although they have different behaviour in the area.
\end{abstract}

\section{KEYWORDS}

Ibera's wetland; Corrientes; network analysis; Alpha and Gamma indexes; Kernel density.

(*) Traducción al inglés por la Prof. Érica Leonor Gómez

Publicado en formato digital: Prof. Federico Carlos Arias, Dr. Osvaldo Daniel Cardozo y Mgter. Miguel Alejandro Parras. ANÁLISIS DE CONECTIVIDAD Y DENSIDAD DE LA RED VIAL EN LA RESERVA NATURAL DEL IBERÁ CON SISTEMAS DE INFORMACIÓN GEOGRÁFICA (SIG). Revista Geográfica Digital. IGUNNE. Facultad de Humanidades. UNNE. Año 13. № 26. Julio - Diciembre 2016. ISSN 1668-5180 Resistencia, Chaco.

En: http://hum.unne.edu.ar/revistas/geoweb/default.htm 
Revista Geográfica Digital. IGUNNE. Facultad de Humanidades. UNNE. Año 13. Nº 26.

Julio - Diciembre 2016. ISSN 1668-5180 Resistencia, Chaco

\section{INTRODUCCIÓN}

El análisis espacial de las infraestructuras de transporte tiene un gran valor en el campo del planeamiento del transporte y del ordenamiento territorial, ya que como lo señala Izquierdo (2001) vertebran el territorio constituyendo un elemento básico para impulsar el desarrollo regional. La presencia de equipamiento e infraestructura vinculada al transporte, tiene una relación recíproca con el espacio geográfico, ya que lo transforman y al mismo tiempo se adaptan a los rasgos fisonómicos del mismo (forma, pendiente, longitud, drenaje, etc.). En este sentido, la Geografía desde hace tiempo ha mostrado interés en analizar estas relaciones y sus resultados sobre el territorio.

Para Potrykowski y Taylor (1984) las redes constituyen un elemento clave para explicar la realidad del territorio, dado que son el principal elemento del Sistema de Transporte que incluye todas las instalaciones técnicas, cuestiones económicas y de gestión, para organizar y ejecutar todo el proceso de transporte. Asimismo, en el análisis espacial, las redes transporte constituyen el sistema arterial de la organización regional y posibilitan la circulación de los flujos, permitiendo articular porciones del territorio (Seguí Pons, 1995).

Hasta la primera mitad del siglo XX los análisis eran de tipo histórico y tecnológico, tanto desde los problemas del territorio como desde las infraestructuras existentes vinculadas al transporte, pero hacia los años 50' la Geografía incorpora técnicas cuantitativas a la tarea de comparar, describir y analizar las diferencias espaciales, por lo que el transporte cobra mayor relevancia en los estudios geográficos con acento en el análisis espacial. En este contexto, la región se explica a través de temas tales como la red de transporte, flujos en las redes, el efecto de las redes en el desarrollo económico (Seguí Pons y Martínez Reynés, 2004).

Desde la óptica del estudio de la estructura espacial de la red, en la década de 1960 comienza el empleo métodos gráfico-geométricos basados en las propiedades topológicas de las redes. Al respecto, la Teoría de Grafos permite asociar una estructura abstracta pero sencilla de nodos conectados por arcos conocida como grafo, a objetos geográficos de la vida real -redes eléctricas, ferroviarias, carreteras, telefónicas, hidrográficas, etc.-. La ventaja de este enfoque reside en la facilidad para el cálculo de un conjunto de índices que miden conectividad, accesibilidad y centralidad en las redes.

Durante los años '70, la perspectiva de análisis se reorienta hacia la interpretación subjetiva de los grupos sociales. El enfoque humanista incluye métodos de análisis cualitativos como la entrevista y la observación directa. En este sentido, el espacio referencial sustituye al espacio abstracto, propio de los trabajos en Geografía de Transporte con enfoque cuantitativo, lo que permite la inclusión de aportes provenientes desde otras disciplinas como la psicología, sociología, etnografía (Seguí Pons y Petrus Bey, 1991), es decir, la atención se centra en estudios de movilidad urbana, de grupos sociales particulares y dando relevancia a las desigualdades que surgen de la distribución de infraestructuras y oportunidades de acceso al servicio de transporte.

La importancia del funcionamiento de las redes ha generado un extenso cuerpo teórico-metodológico, a partir del cual se desprenden numerosas técnicas y procedimientos para su estudio. Se considera oportuno insertar el uso de los Sistemas de Información Geográfica -SIG- como un campo transversal, que contribuye con numerosas herramienta para la generación de nuevos datos (tasas, índices, etc.), para el análisis y modelado de variables, así como para la elaboración de cartografía. Moldes Teo (1995) reconoce un amplio espectro de aplicaciones de los SIG en el campo de los transportes y su organización, afirmando que constituye un eje tecnológico fundamental capaz de realizar aportes significativos a partir de su planificación, optimización y gestión principal para la mejora y empleo eficiente de los sistemas de transporte.

En la actualidad, la mayoría de los SIG tanto comerciales como libres, han desarrollado varias herramientas destinadas a la construcción de redes con ajuste topológico, áreas de captura de la

Publicado en formato digital: Prof. Federico Carlos Arias, Dr. Osvaldo Daniel Cardozo y Mgter. Miguel Alejandro Parras. ANÁLISIS DE CONECTIVIDAD Y DENSIDAD DE LA RED VIAL EN LA RESERVA NATURAL DEL IBERÁ CON SISTEMAS DE INFORMACIÓN GEOGRÁFICA (SIG). Revista Geográfica Digital. IGUNNE. Facultad de Humanidades. UNNE. Año 13. N N 26. Julio - Diciembre 2016. ISSN 1668-5180 Resistencia, Chaco.

En: http://hum.unne.edu.ar/revistas/geoweb/default.htm 
demanda u oferta -buffer, service area, voronoi-, flujos ponderados entre origen y destino, estimación de densidades, así como los clásicos problemas de ruteo que simulan desplazamientos buscando el camino más corto a uno o varios destinos. Muchos organismos de la administración pública y empresas han incorporado estas funciones a la resolución problemas en sus tareas de rutina.

Una ventaja inherente al uso de los SIG para el análisis de cuestiones territoriales, es la gran versatilidad que presenta para el manejo de los datos a diferentes escalas. Esta propiedad permitió seleccionar las técnicas de análisis más apropiadas para el área de interés: los Esteros del Iberá en la Provincia de Corrientes.

El área de estudio corresponde a la Reserva Natural de los Esteros de Iberá. Se trata de un humedal ubicado en el centro-norte de la Provincia (ver Figura 1) con una extensión aproximada de 1.300.000 ha, que constituye una de las áreas naturales y reservorios de agua dulce más importantes de Sudamérica.

En términos generales, el Iberá constituye una cuenca alimentada básicamente por aportes pluviales unos $1.200 \mathrm{~mm}$ anuales-, que debido al lento escurrimiento de las aguas por la escasa pendiente del terreno se mantiene anegada, dando lugar a la presencia de numerosos esteros, lagunas, bañados y cursos fluviales quienes lentamente drenan sus aguas en dirección noreste-sudoeste hacia el río Corriente. En las áreas inundadas se desarrollan extensos pajonales, totorales y juncales, en los albardones hay sauces y ceibos, mientras que en las lomadas se desarrollan pastizales y monte (Manoiloff y Rey, 2005).

\section{Figura 1: Localización geográfica de la Reserva Natural Esteros del Iberá}

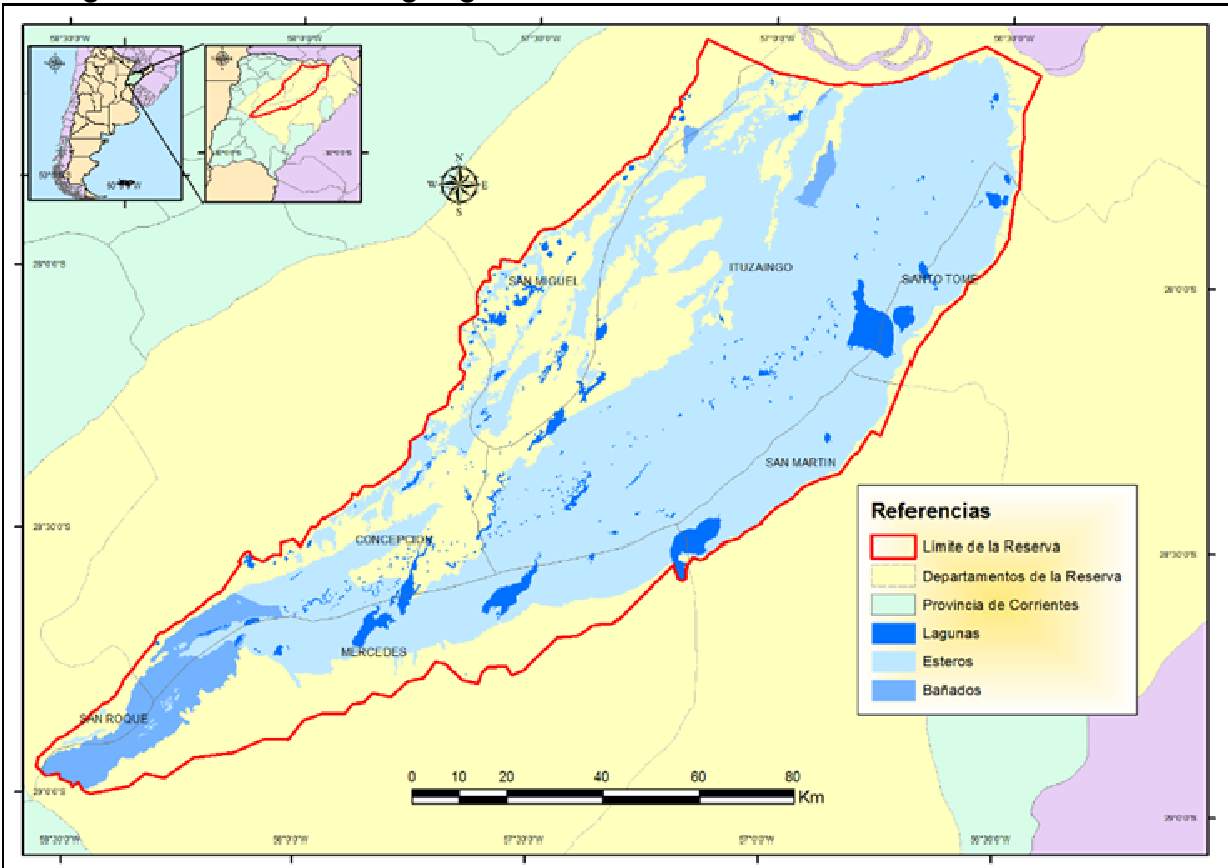

Fuente: Elaboración propia en base a los datos proporcionados por el Instituto Geográfico Nacional.

Desde el punto de vista demográfico se trata de un espacio escasamente poblado. Pese a que incluye siete departamentos (Ituzaingó, San Miguel, Concepción, San Roque, Mercedes, General San Martín y Santo Tomé) y unos 27 municipios, la población en ellos no supera los 200.000 hab. de acuerdo al último censo (INDEC, 2010). Esto representa apenas el $20 \%$ del total provincial, proporción aún más reducida

Publicado en formato digital: Prof. Federico Carlos Arias, Dr. Osvaldo Daniel Cardozo y Mgter. Miguel Alejandro Parras. ANÁLISIS DE CONECTIVIDAD Y DENSIDAD DE LA RED VIAL EN LA RESERVA NATURAL DEL IBERÁ CON SISTEMAS DE INFORMACIÓN GEOGRÁFICA (SIG). Revista Geográfica Digital. IGUNNE. Facultad de Humanidades. UNNE. Año 13. № 26. Julio - Diciembre 2016. ISSN 1668-5180 Resistencia, Chaco. 
Revista Geográfica Digital. IGUNNE. Facultad de Humanidades. UNNE. Año 13. № 26.

Julio - Diciembre 2016. ISSN 1668-5180 Resistencia, Chaco

si tenemos en cuenta que buena parte de ella reside en los centros urbanos localizados de su periferia (San Miguel, Concepción, Ituzaingó, Mercedes, Loreto, etc).

El área de la reserva dispone de un gran número de estudios referidos a sus características naturales fauna y flora en sus diversas formas, limnología, geomorfología, pedología, etc.-, sin embargo es escasa la información existente sobre las características humanas, y menos aún trabajos que aborden el estudio de las infraestructuras existentes, como las de transporte.

Una revisión de la documentación existente -cartográfica sobre todo-, permite advertir claros desequilibrios en la distribución y configuración espacial de la red vial en la Reserva. Esto conduce a formular la hipótesis de que dicha configuración espacial genera una baja cohesión territorial producto de la escasa densidad y conectividad vial, con tanto interna como hacia el resto de la Provincia.

No existen antecedentes específicos sobre la cuestión en el área de estudio, pero a escala provincial. la Geográfica № 12 (Manoiloff y Rey, 2004) representa 8 planchas cartográficas con información referida al transporte en la Provincia, donde se destacan las referidas a densidad de vías, accesibilidad y frecuencia del tránsito medio diario; en esas representaciones queda en evidencia el "vacío" correspondiente a los Esteros del Iberá. Por otra parte, Insaurralde y Cardozo (2010) efectúan un análisis de conectividad y accesibilidad sobre la red primaria pavimentada, remarcando que esa configuración evita la comunicación directa entre el este y oeste de la Provincia.

Atendiendo a esta problemática, el objetivo general del trabajo es contribuir al estudio de la configuración espacial que adquiere la red vial al interior de la Reserva del lberá, buscando poner en evidencia desequilibrios en su trazado.

En particular, se busca conocer el grado de conectividad de la red por medio de algunos índices propios de la Teoría de Grafos, como así también, identificar patrones espaciales que surjan del cálculo de la densidad kernel.

Debe mencionarse que los resultados de este trabajo son producto de una Beca de Investigación en categoría Pregrado, otorgada en 2014 por la Secretaria General de Ciencia y Técnica (SGCyT) de la Universidad Nacional del Nordeste (UNNE), desarrollada en el marco del PI 12lH01 "La población y su territorio. La acción antrópica en la configuración territorial del Iberá (Corrientes, Argentina)", financiado por la SGCyT de nuestra Universidad para el periodo 2013-2015.

\section{MATERIALES Y MÉTODOS}

Cabe señalar que buena parte de la integración y representación de los datos se basó en el uso de información digital georreferenciada -en formato ESRI shapefile-, aprovechando la fuerte capacidad analítica y de procesamiento que tienen los SIG.

Una de las primeras tareas metodológicas consistió en la obtención de datos gráficos y alfanuméricos. Para ello se consultó el servidor web del Instituto Geográfico Nacional (IGN), donde se obtuvo información georreferenciada de los cuerpos y cursos de aguas, límites -nacional, provincial, y departamental-, localidades y red vial. Por otra parte, los límites de la Reserva Natural corresponden a la Dirección General de Catastro y Cartografía (DGCyC) de la Provincia de Corrientes, mientras que los datos demográficos se obtuvieron de la Dirección de Estadística (DEyC) y Censos provincial. Además, en el marco del PI 12IH01, se digitalizó una versión mejorada de la red vial interna con corrección de tramos y control topológico, debido a que la proveniente del IGN no tenía el grado de precisión y actualización requerida.

Una vez reunida la información geográfica se procedió a uniformar la referencia espacial, adoptando un sistemas de coordenadas planas y Posgar 98 como marco de referencia espacial, lo cual permite operar en un SIG con la información superpuesta en forma de capas, algo fundamental para el posterior procesamiento de los datos y elaboración cartográfica.

Publicado en formato digital: Prof. Federico Carlos Arias, Dr. Osvaldo Daniel Cardozo y Mgter. Miguel Alejandro Parras. ANÁLISIS DE CONECTIVIDAD Y DENSIDAD DE LA RED VIAL EN LA RESERVA NATURAL DEL IBERÁ CON SISTEMAS DE INFORMACIÓN GEOGRÁFICA (SIG). Revista Geográfica Digital. IGUNNE. Facultad de Humanidades. UNNE. Año 13. N No 26. Julio - Diciembre 2016. ISSN 1668-5180 Resistencia, Chaco.

En: http://hum.unne.edu.ar/revistas/geoweb/default.htm 
Revista Geográfica Digital. IGUNNE. Facultad de Humanidades. UNNE. Año 13. Nº 26.

Julio - Diciembre 2016. ISSN 1668-5180 Resistencia, Chaco

Los primeros tratamientos de la información geográfica en un entorno SIG tuvieron que ver con operaciones sencillas como:

- Select by Location: para seleccionar la red vial y departamento contenidos dentro de la Reserva.

- Select by Attributes: para seleccionar las localidades de los departamentos incluidos en la Reserva.

- Clip: para recortar los tramos de red vial incluidas dentro del área de la Reserva.

- Spatial Join: para agregar el nombre del departamento a cada tramo de la red vial.

- Dissolve: para agregar los tramos de red vial por departamento para obtener los totales de longitud.

- Calculate Geometry: para actualizar las longitudes de los tramos de red vial, luego de ser cortados o actualizados.

La aplicación de dichos geoprocesos permitió el cálculo de algunas variables para conocer medidas reales de la red vial: longitud en kilómetros según departamento, participación hacia al interior de la Reserva, según el material de la calzada -pavimento, consolidado, de tierra-, etc.

Por otra parte, desde la Teoría de Grafos que adopta una perspectiva de análisis abstracta basada en las propiedades topológicas de una red, fueron seleccionadas dos medidas de conexión global (Alfa y Gamma) para describir la red vial dentro de la Reserva.

1. Índice Gamma: es el cociente entre el número de arcos presentes en una red y el máximo número posible; permite la comparación de distintas redes, y sus valores van entre 0 y 1 para una red totalmente desconectada y conectada respectivamente. Su ecuación es la siguiente:

$$
\gamma=a / a_{\max }
$$

donde: $\gamma$ es el valor obtenido para Gamma

$a$ es el número de arcos que tiene en el grafo

$a_{\max }$ es el número máximo de arcos que puede tener el grafo

2. Índice Alfa: es el cociente entre el número real de ciclos en una red y el máximo número posible; los valores varían entre 0 para un grafo sin ningún circuito y 1 para un grafo completo, por lo tanto valores altos indican la presencia de una red más sólida. Su ecuación es la siguiente:

$$
\alpha=\mu / 2 \cdot n-5
$$

donde: $\alpha$ es el valor obtenido para Alfa

$\mu$ es el número de circuitos observados en el grafo

$2 \cdot n-5$ es el máximo número posible

En esta etapa se recurrió nuevamente a los SIG donde, sobre la extensión Network Analysis de ArcView 3.x se ejecuta un script programado por Wong and Lee (2005) en lenguaje Aveneu, para calcular ambos índices y visualizar su resultado en el territorio.

Para definir con precisión la distribución espacial de los datos se presenta el inconveniente de que, por lo general las distribuciones son heterogéneas -con clúster y vacíos-, además del límite impuesto por las unidades administrativas que tiene una gran influencia sobre el cálculo según la superficie que abarque. Esto puede solucionarse con métodos que describen la intensidad en la presencia de un fenómeno, y entre ellos se encuentran el cálculo de la densidad.

Publicado en formato digital: Prof. Federico Carlos Arias, Dr. Osvaldo Daniel Cardozo y Mgter. Miguel Alejandro Parras. ANÁLISIS DE CONECTIVIDAD Y DENSIDAD DE LA RED VIAL EN LA RESERVA NATURAL DEL IBERÁ CON SISTEMAS DE INFORMACIÓN GEOGRÁFICA (SIG). Revista Geográfica Digital. IGUNNE. Facultad de Humanidades. UNNE. Año 13. № 26. Julio - Diciembre 2016. ISSN 1668-5180 Resistencia, Chaco.

En: http://hum.unne.edu.ar/revistas/geoweb/default.htm 
Frente a los clásicos mapas de densidad que adoptan unidades administrativas como denominador en el cálculo, se propone utilizar aquí un método de densidad local basado en un modelo de datos raster. Se trata de definir para cada píxel un valor de densidad obtenido como resultado del cociente entre, las observaciones próximas definidas por un entorno de tipo circular y la superficie definida por el mismo (Moreno Jiménez, 2008).

En el caso de la densidad focal o Kernel, incorpora además una función de ponderación tipo exponencial al momento de asignar el valor a cada píxel, de manera que las observaciones más cercanas al centro del pixel tengan un mayor peso en el cálculo y las lejanas una menor influencia. El resultado es una superficie continua y suave que denota mejor los cambios en las distribuciones sobre el espacio.

\section{RESULTADOS}

La red vial incluida dentro de la Reserva presenta una distribución heterogenea, en clara correspondencia con las limitaciones impuestas por el medio natural, pero este comportamiento varía de acuerdo a la fuente de datos empleada, ya que existe una diferencia notable en la escala de trabajo. En este sentido, el Instituto Geográfico Nacional emplea las cartas toporáficas de escala en 1:250.000, donde la red tiene una mayor extension aparente hacia el noroeste y sur del área, es decir, en los departamentos de Ituzaingó, San Miguel y Mercedes, siendo nula en la zona central del mismo (ver Figura 2).

Figura 2: Red Vial de la Reserva Natural Esteros del Iberá (IGN)

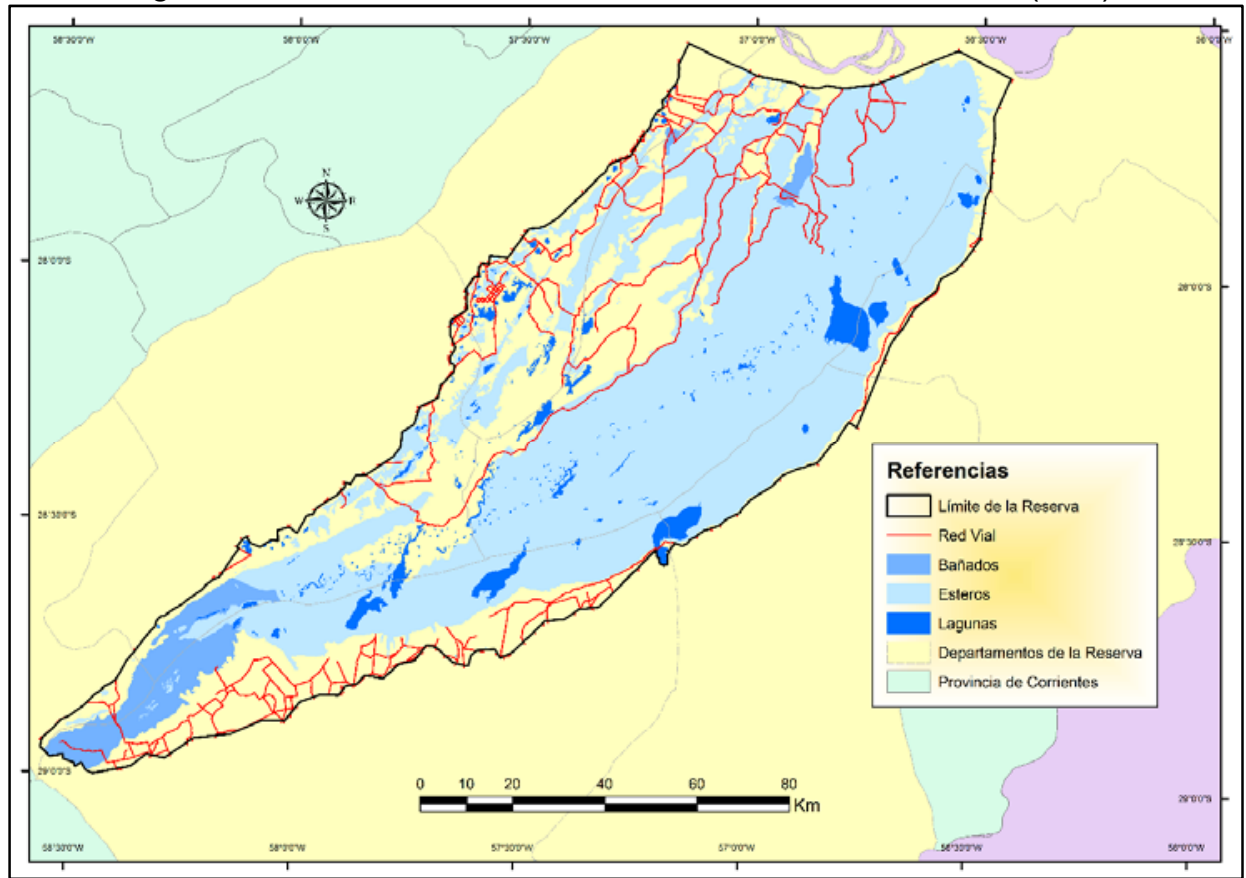

Fuente: Elaboración propia en base a los datos proporcionados por el Instituto Geográfico Nacional.

En la misma linea, a partir del proyecto mencionado con anterioridad, se realizó una digitalización de la red vial a escala 1:25.000, cuya distribución presenta algunas diferencias de la proporcionada por el IGN. En principio se mantiene la mayor extension de red para los departamentos Ituzaingó, San Miguel y Mercedes, pero se incorporan los departamentos de Concepción, San Roque y San Martin, continuando el gran vacío del área central, incluyendo amplios sectores de diferentes departamentos, en especial Itizaingó (ver Figura 3).

Publicado en formato digital: Prof. Federico Carlos Arias, Dr. Osvaldo Daniel Cardozo y Mgter. Miguel Alejandro Parras. ANÁLISIS DE CONECTIVIDAD Y DENSIDAD DE LA RED VIAL EN LA RESERVA NATURAL DEL IBERÁ CON SISTEMAS DE INFORMACIÓN GEOGRÁFICA (SIG). Revista Geográfica Digital. IGUNNE. Facultad de Humanidades. UNNE. Año 13. N N 26. Julio - Diciembre 2016. ISSN 1668-5180 Resistencia, Chaco. 
Revista Geográfica Digital. IGUNNE. Facultad de Humanidades. UNNE. Año 13. № 26. Julio - Diciembre 2016. ISSN 1668-5180 Resistencia, Chaco

Figura 3: Red Vial de la Reserva Natural Esteros del Iberá (PI 12IH01)

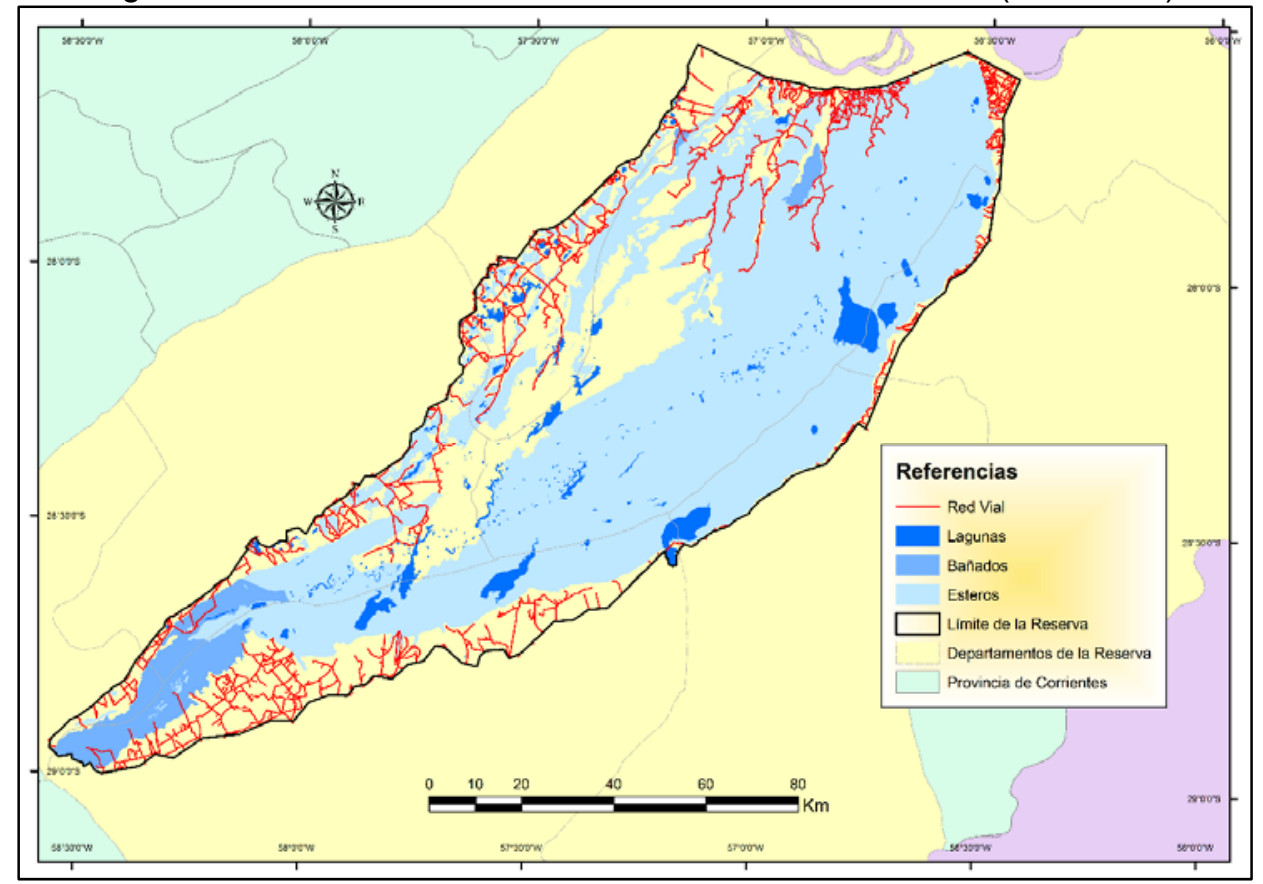

Fuente: Elaboración propia en base a los datos proporcionados por la Dirección Provincial de Vialidad de Corrientes y digitalización propia por PI $12 \mathrm{IH} 01$.

Por otro lado, respecto a la longitud total de la red en el área de estudio, existen diferencias conciderables respecto a la extensión, como en la participación por departamentos. En la Tabla 1(a) se representan los valores correspondiente a la extensión de la red vial por departamento a partir de los datos proporcionados por el IGN, Ituzaingó posee la mayor extension con $702,32 \mathrm{~km}$ y San Roque la menor con 48,27 km. Respecto a la red digitalizada, en la Tabla 1(b) si bien se mantienen los mismos departamentos con máxima y mínima longitud, los valores aumentan, siendo 979,05 km para Intuzaingó y $82,66 \mathrm{~km}$ para San Roque; otra diferencia notable se da en la longitud total de las redes, ya que en el caso de la red generada con datos del proyecto PI 12l 01 , cuenta con aproximadamente un $50 \%$ mas que la red IGN.

Ahora bien, a fines de comparar la participación relativa de cada departamento en el total, se calcularon los valores relativos expresados en porcencentaje. Un dato sobresaliente que arroja la comparación es que en ambos casos -IGN y PI 12IH01-, los departamentos de Ituzaingó y Mercedes concentran cerca del $60 \%$ del total de la red, mientras que Santo Tomé, San Martín y San Roque en conjunto apenas superan el $10 \%$ de participación, lo que refleja claramente el desequilibrio existente dentro de la Reserva.

Tabla 1: Longitud de la red vial en el área de la Reserva Natural Esteros del Iberá, por departamento

\begin{tabular}{|l|c|c|c|c|}
\hline DEPARTAMENTOS & $\begin{array}{c}\text { (a) RED INTERIOR } \\
\text { DE LA RESERVA } \\
\text { IGN (KM) }\end{array}$ & $\begin{array}{c}\text { (a1) PARTICIPACIÓN } \\
\text { \% DE RED IGN }\end{array}$ & $\begin{array}{c}\text { (b) RED INTERIOR } \\
\text { DE LA RESERVA } \\
\text { PI12IH01 (KM) }\end{array}$ & $\begin{array}{c}\text { (b1) PARTICIPACIÓN } \\
\text { \% DE RED PI12IH01 }\end{array}$ \\
\hline Concepción & 182,43 & 9,3 & 447,08 & 15,1 \\
\hline Ituzaingó & 702,32 & 35,7 & 979,05 & 33,1 \\
\hline
\end{tabular}

Publicado en formato digital: Prof. Federico Carlos Arias, Dr. Osvaldo Daniel Cardozo y Mgter. Miguel Alejandro Parras. ANÁLISIS DE CONECTIVIDAD Y DENSIDAD DE LA RED VIAL EN LA RESERVA NATURAL DEL IBERÁ CON SISTEMAS DE INFORMACIÓN GEOGRÁFICA (SIG). Revista Geográfica Digital. IGUNNE. Facultad de Humanidades. UNNE. Año 13. № 26. Julio - Diciembre 2016. ISSN 1668-5180 Resistencia, Chaco.

En: http://hum.unne.edu.ar/revistas/geoweb/default.htm 


\begin{tabular}{|l|r|r|r|r|} 
Mercedes & 490,41 & 24,9 & 688,91 & 23,3 \\
\hline San Martín & 78,59 & 4,0 & 107,49 & 3,6 \\
\hline San Miguel & 382,98 & 19,4 & 529,37 & 17,9 \\
\hline San Roque & 48,27 & 2,5 & 82,66 & 2,8 \\
\hline Santo Tomé & 84,42 & 4,3 & 126,76 & 4,3 \\
\hline Total & $\mathbf{1 9 6 9 , 4 2}$ & $\mathbf{1 0 0}$ & $\mathbf{2 9 6 1 , 3 2}$ & $\mathbf{1 0 0}$ \\
\hline
\end{tabular}

Fuente: Elaboración propia en base a los datos proporcionados por Instituto Geográfico Nacional, la Dirección Provincial de Vialidad de Corrientes y digitalización propia por PI 12IH01.

Otra cuestión importante de analizar porque suele estar asociada a la accesibilidad en el territorio, es el cálculo de densidad hacia el interior de la red. En la Figura 4 se representa la densidad a partir de los datos del IGN, en ella se advierte la presencia de mayor densidad en San Miguel, Ituzaingó y Mercedes, con núcleos de alta densidad en las zonas cercanas a las ciudades cabeceras, con valores que oscilan entre 0,44 y $0,55 \mathrm{~km} / \mathrm{km}^{2}$ de red y un fuerte vacío en la zona central en sentido noreste-sudoeste, básicamente por no tener de ningún tipo de red sobre ese sector.

Figura 4: Densidad de la red vial en el área de la Reserva Natural Esteros del Iberá (IGN)

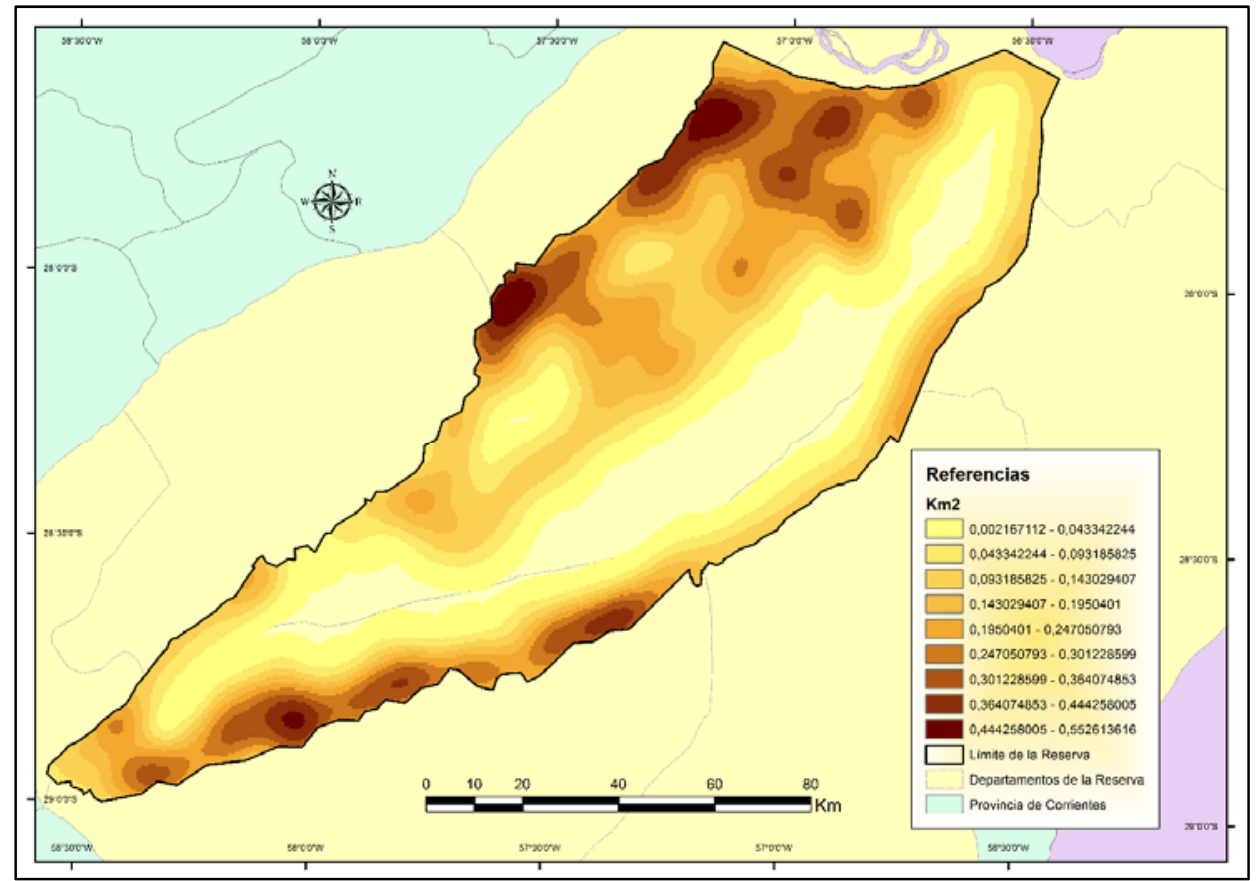

Fuente: Elaboración propia en base a los datos proporcionados por el Instituto Geográfico Nacional.

En el caso de la Figura 5, la situación cambia significativamente, ya que los mayores valores -oscilan entre 1,04 y $1,31 \mathrm{~km} / \mathrm{km}^{2}$ - están situados en el departamento Ituzaingó y otros núcleos secundarios en San Miguel y Mercedes -valores de 0,62 a $0,81 \mathrm{~km} / \mathrm{km}^{2}$-, mientras que el vacío sigue ocupando el área central, al no tener presencia de red como en el caso anterior.

Figura 5: Densidad de la red vial en el área de la Reserva Natural Esteros del Iberá (PI 12IH01)

Publicado en formato digital: Prof. Federico Carlos Arias, Dr. Osvaldo Daniel Cardozo y Mgter. Miguel Alejandro Parras. ANÁLISIS DE CONECTIVIDAD Y DENSIDAD DE LA RED VIAL EN LA RESERVA NATURAL DEL IBERÁ CON SISTEMAS DE INFORMACIÓN GEOGRÁFICA (SIG). Revista Geográfica Digital. IGUNNE. Facultad de Humanidades. UNNE. Año 13. № 26. Julio - Diciembre 2016. ISSN 1668-5180 Resistencia, Chaco.

En: http://hum.unne.edu.ar/revistas/geoweb/default.htm 


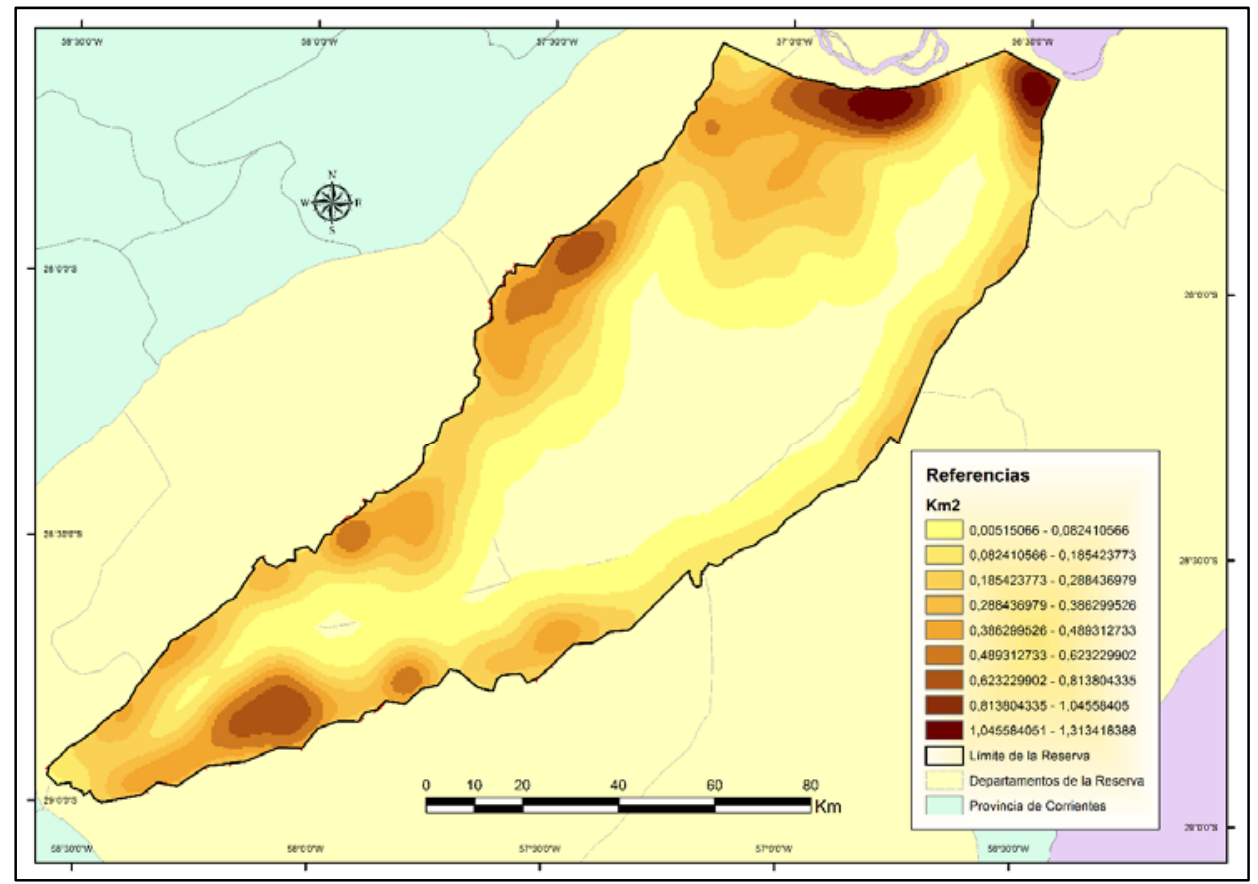

Fuente: Elaboración propia en base a los datos proporcionados por Instituto Geográfico Nacional, la Dirección Provincial de Vialidad de Corrientes y digitalización propia por PI 12IH01.

Hasta el momento se presentó una descripción de la distribución de la red vial por medio de la densidad, como así también la extensión, participación y densidad de los departamentos que la conforman hacia su interior.

Sin embargo, la red puede ser interpretada como un grafo a partir de una sencilla estructura de tipo arconodos, donde se pueden realizar multiplicidad de relaciones y análisis para evaluar la red. En este caso, se emplean medidas de conectividad para ver el grado de conexión existente, comparando nuevamente el comportamiento dependiendo la fuente y escala de relevamiento.

Los índices empleados en esta oportunidad para el análisis de conectividad son dos:

1. Índice Alfa, mide las posibilidades de relaciones alternativas que ofrece la red, a partir del número de circuitos observados en el grafo y los que existirían en caso de tratarse de un grafo completo.

2. Índice Gamma, mide las posibilidades de relaciones directas que ofrece la red a partir del número de arcos existentes y el máximo número que pueda existir en el grafo, considerando la cantidad de nodos existentes.

Figura 6: Representación Cartográfica del índice Alfa por departamentos en el área de la Reserva Natural Esteros del Iberá (IGN)

Publicado en formato digital: Prof. Federico Carlos Arias, Dr. Osvaldo Daniel Cardozo y Mgter. Miguel Alejandro Parras. ANÁLISIS DE CONECTIVIDAD Y DENSIDAD DE LA RED VIAL EN LA RESERVA NATURAL DEL IBERÁ CON SISTEMAS DE INFORMACIÓN GEOGRÁFICA (SIG). Revista Geográfica Digital. IGUNNE. Facultad de Humanidades. UNNE. Año 13. № 26. Julio - Diciembre 2016. ISSN 1668-5180 Resistencia, Chaco.

En: http://hum.unne.edu.ar/revistas/geoweb/default.htm 
Revista Geográfica Digital. IGUNNE. Facultad de Humanidades. UNNE. Año 13. № 26. Julio - Diciembre 2016. ISSN 1668-5180 Resistencia, Chaco

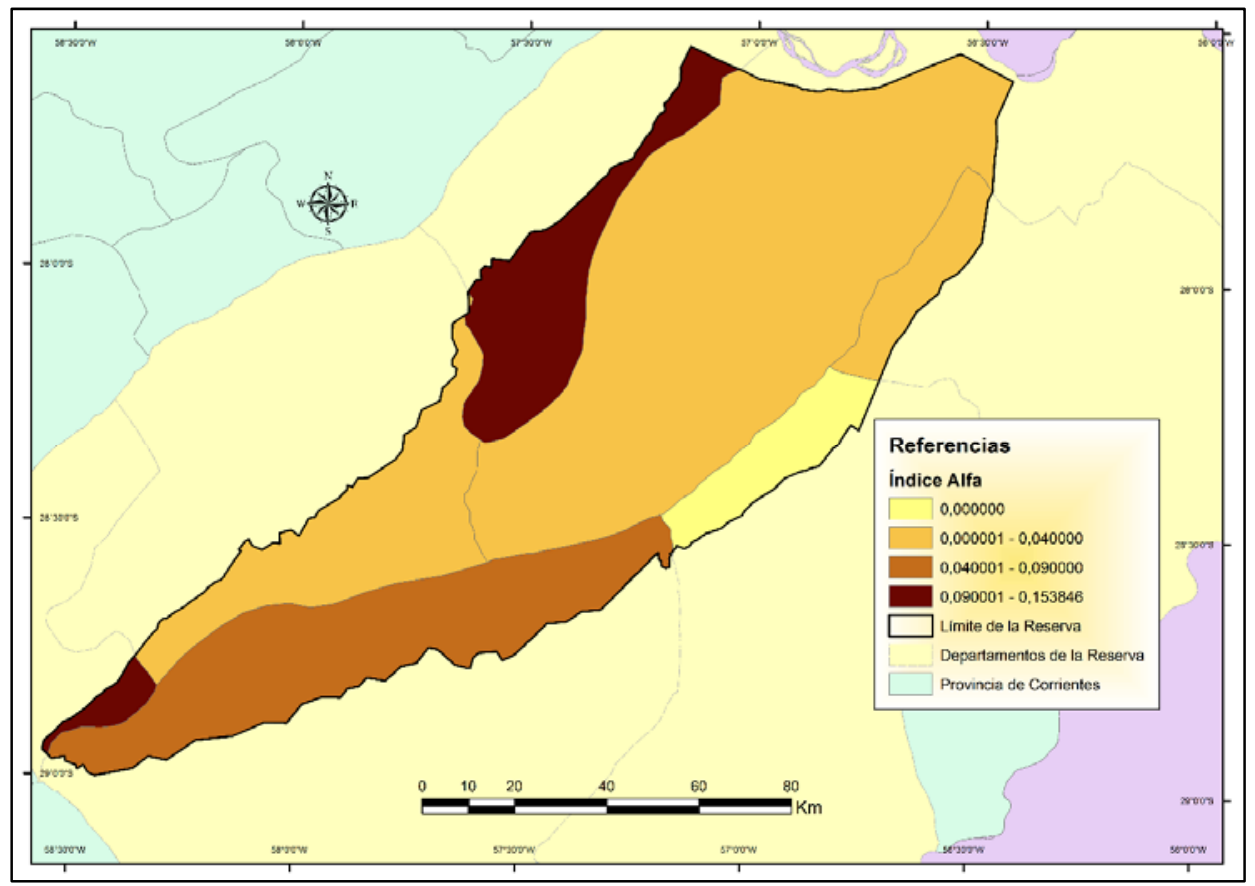

Fuente: Elaboración propia en base a los datos proporcionados por el Instituto Geográfico Nacional.

Al aplicar cado uno de los índices a la red vial y según las fuente de información considerada, se advierte que en el caso del índice Alfa existe una variacion considerable entre los valores calculados. En la Figura 6 los valores son relativamente bajos para todos los departamento, porque son inferiores a 0,2, no obstante se denota que en San Roque y San Miguiel estan los grafos con mayor cantidad de circuitos observados, con valores de 0,153 y 0,098 respectivamente, mientras que en San Martín se da un valor de 0 , es decir, ningún circuito presente en la red (ver Tabla 2a).

Tabla 2: Resumen de medidas topológicas de grafos (Índice Alfa) en el área de la Reserva Natural Esteros del Iberá, por departamento

\begin{tabular}{|l|r|r|r|r|r|r|}
\hline \multirow{2}{*}{ DEPARTAMENTOS } & \multicolumn{3}{|c|}{ (a) Red Vial IGN } & \multicolumn{3}{c|}{ (b) Red Vial PI 12IH01 } \\
\cline { 2 - 7 } & VÉRTICES & ARCOS & ÍNDICE ALFA & VÉRTICES & \multicolumn{1}{c|}{ ARCOS } & ÍNDICE ALFA \\
\hline Concepción & 53 & 48 & 0,039604 & 203 & 211 & 0,02244 \\
\hline Ituzaingó & 119 & 126 & 0,034334 & 776 & 934 & 0,10278 \\
\hline Mercedes & 121 & 139 & 0,080168 & 455 & 435 & 0,02099 \\
\hline San Martín & 19 & 18 & 0 & 118 & 121 & 0,01731 \\
\hline San Miguel & 145 & 172 & 0,098245 & 309 & 343 & 0,05709 \\
\hline San Roque & 9 & 10 & 0,153846 & 48 & 47 & 0 \\
\hline Santo Tomé & 26 & 26 & 0,021276 & 141 & 158 & 0,06498 \\
\hline \multicolumn{1}{|c|}{ Total } & $\mathbf{4 9 2}$ & $\mathbf{5 3 9}$ & $\mathbf{0 , 0 8 4 5 7 1}$ & $\mathbf{2 0 5 0}$ & $\mathbf{2 2 4 9}$ & $\mathbf{0 , 0 5 4 4 9}$ \\
\hline
\end{tabular}

Fuente: Elaboración propia en base a los datos proporcionados por Instituto Geográfico Nacional, la Dirección Provincial de Vialidad de Corrientes y digitalización propia por PI $12 \mathrm{IH} 01$.

Desde una perspectiva general, en la Figura 7 se mantienen valores poco significativos en el índice Alfa, pero si nos observamos en la manera en que distribuye, la situación cambia respecto al caso anterior, donde Ituzaingó posee el mayor valor con un índice alfa de 0,10278 y San Roque el menor valor (ver Tabla 2b).

Publicado en formato digital: Prof. Federico Carlos Arias, Dr. Osvaldo Daniel Cardozo y Mgter. Miguel Alejandro Parras. ANÁLISIS DE CONECTIVIDAD Y DENSIDAD DE LA RED VIAL EN LA RESERVA NATURAL DEL IBERÁ CON SISTEMAS DE INFORMACIÓN GEOGRÁFICA (SIG). Revista Geográfica Digital. IGUNNE. Facultad de Humanidades. UNNE. Año 13. N N 26. Julio - Diciembre 2016. ISSN 1668-5180 Resistencia, Chaco.

En: http://hum.unne.edu.ar/revistas/geoweb/default.htm 
Figura 7: Representación Cartográfica del índice Alfa por departamentos en el área de la Reserva Natural Esteros del Iberá (PI 12IH01)

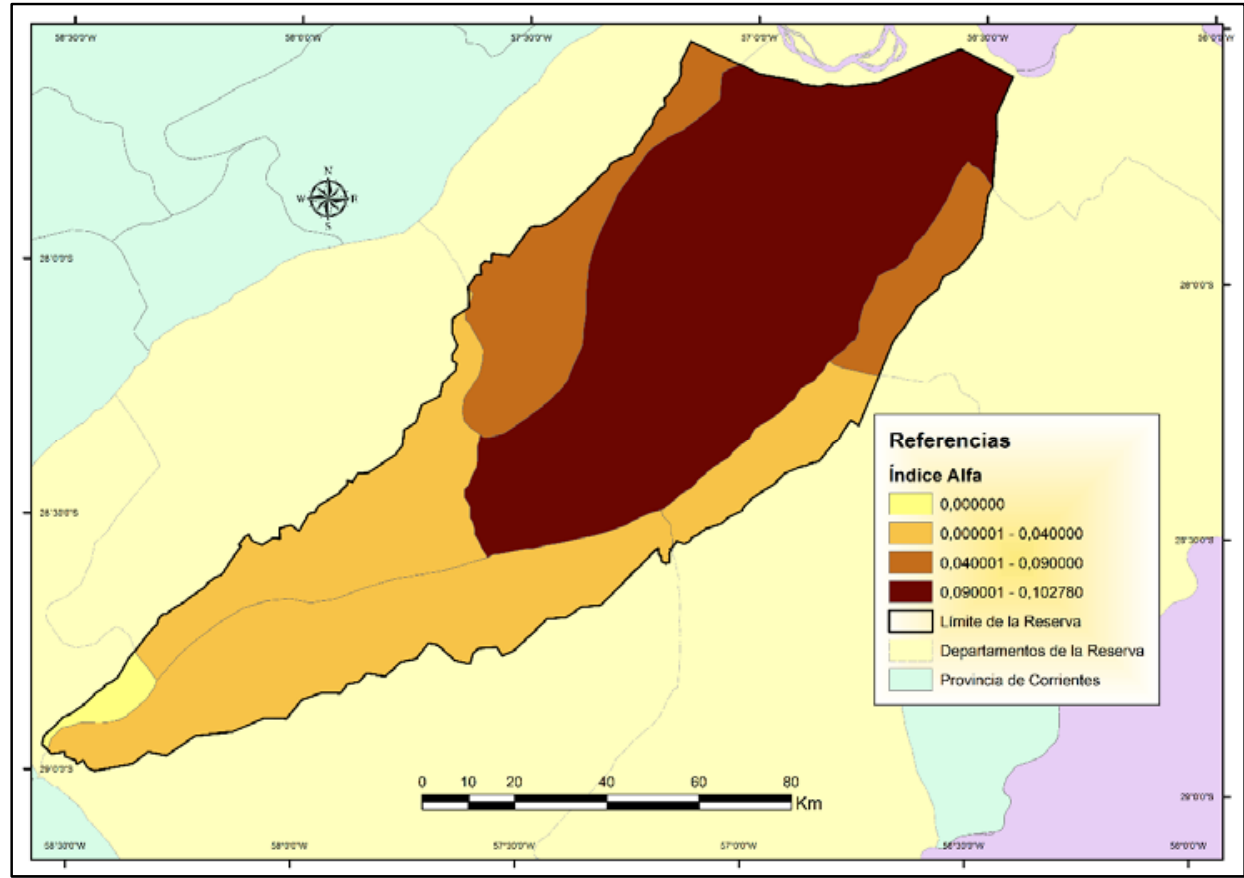

Fuente: Elaboración propia en base a los datos proporcionados por la Dirección Provincial de Vialidad de Corrientes y digitalización propia por PI $12 \mathrm{IH} 01$.

Por otro lado, para evidenciar las relaciones directas dentro de la red a partir de los nodos existentes y potenciales, se calculó el índice Gamma. En este sentido, en la Figura 8 se observa en general valores que si bien son inferiores a 0,5 , no son despreciables, debido a las particularidades del espacio desde el punto de vista natural. San Roque y San Miguel son los departamentos con mayor conexión con valores de 0,47619 y 0,400932 respectivamente (ver Tabla 3a).

Figura 8: Representación Cartográfica del índice Gamma por departamentos en el área de la Reserva Natural Esteros del Iberá (IGN)

Publicado en formato digital: Prof. Federico Carlos Arias, Dr. Osvaldo Daniel Cardozo y Mgter. Miguel Alejandro Parras. ANÁLISIS DE CONECTIVIDAD Y DENSIDAD DE LA RED VIAL EN LA RESERVA NATURAL DEL IBERÁ CON SISTEMAS DE INFORMACIÓN GEOGRÁFICA (SIG). Revista Geográfica Digital. IGUNNE. Facultad de Humanidades. UNNE. Año 13. № 26. Julio - Diciembre 2016. ISSN 1668-5180 Resistencia, Chaco.

En: http://hum.unne.edu.ar/revistas/geoweb/default.htm 
Revista Geográfica Digital. IGUNNE. Facultad de Humanidades. UNNE. Año 13. № 26. Julio - Diciembre 2016. ISSN 1668-5180 Resistencia, Chaco

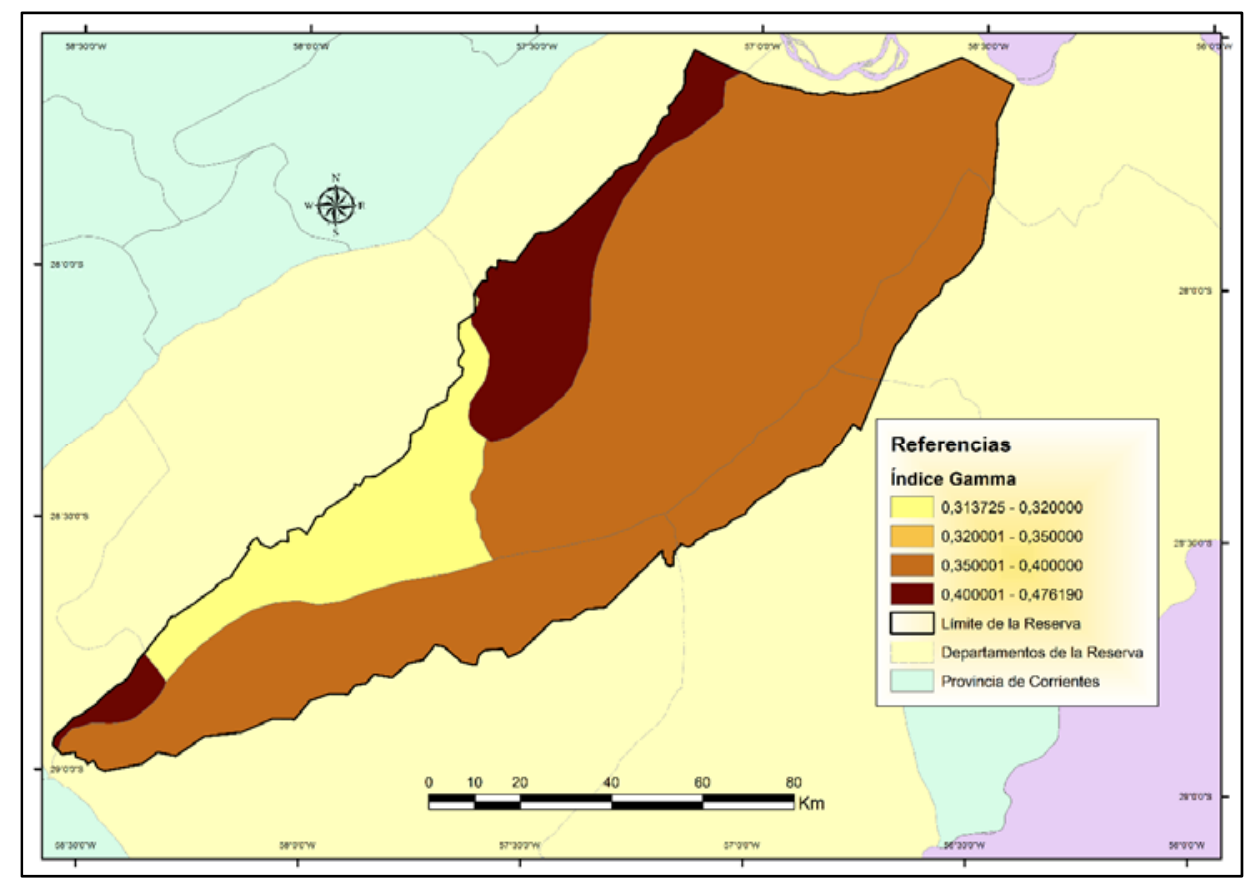

Fuente: Elaboración propia en base a los datos proporcionados por el Instituto Geográfico Nacional.

Tabla 3: Resumen de medidas topológicas de grafos (Índice Gamma) en el área de la Reserva Natural Esteros del Iberá, por departamento

\begin{tabular}{|l|r|r|r|r|r|r|}
\hline & \multicolumn{3}{|c|}{ (a) Red Vial IGN } & \multicolumn{3}{c|}{ (b) Red Vial PI 12IH01 } \\
\hline DEPARTAMENTOS & \multirow{2}{*}{ VÉRTICES } & ARCOS & $\begin{array}{c}\text { ÍNDICE } \\
\text { GAMMA }\end{array}$ & VÉRTICES & ARCOS & $\begin{array}{c}\text { ÍNDICE } \\
\text { GAMMA }\end{array}$ \\
\hline Concepción & 53 & 48 & 0,31372 & 203 & 211 & 0,34991 \\
\hline Ituzaingó & 119 & 126 & 0,35897 & 776 & 934 & 0,40223 \\
\hline Mercedes & 121 & 139 & 0,38935 & 455 & 435 & 0,32008 \\
\hline San Martín & 19 & 18 & 0,35294 & 118 & 121 & 0,34770 \\
\hline San Miguel & 145 & 172 & 0,40093 & 309 & 343 & 0,37242 \\
\hline San Roque & 9 & 10 & 0,47619 & 48 & 47 & 0,34058 \\
\hline Santo Tomé & 26 & 26 & 0,36111 & 141 & 158 & 0,37889 \\
\hline Total & $\mathbf{4 9 2}$ & $\mathbf{5 3 9}$ & $\mathbf{0 , 3 9 0 4 1}$ & $\mathbf{2 0 5 0}$ & $\mathbf{2 2 4 9}$ & $\mathbf{0 , 3 6 9 8 1}$ \\
\hline
\end{tabular}

Fuente: Elaboración propia en base a los datos proporcionados por Instituto Geográfico Nacional, la Dirección Provincial de Vialidad de Corrientes y digitalización propia por PI $12 \mathrm{lH} 01$.

En cuanto a la Figura 9, muestra que la situación general se conserva, es decir, valores inferiores al 0,50, pero la influencia de cada departamento es diferente, ya que Ituzaingó con un 0,402 es quien tiene el mayor índice y Mercedes con un valor de 0,32 es el departamento con menor conexión dentro del área (ver Tabla 3b).

Figura 9: Representación Cartográfica del índice Gamma por departamentos en el área de la Reserva Natural Esteros del Iberá (PI 12IH01)

Publicado en formato digital: Prof. Federico Carlos Arias, Dr. Osvaldo Daniel Cardozo y Mgter. Miguel Alejandro Parras. ANÁLISIS DE CONECTIVIDAD Y DENSIDAD DE LA RED VIAL EN LA RESERVA NATURAL DEL IBERÁ CON SISTEMAS DE INFORMACIÓN GEOGRÁFICA (SIG). Revista Geográfica Digital. IGUNNE. Facultad de Humanidades. UNNE. Año 13. N N 26. Julio - Diciembre 2016. ISSN 1668-5180 Resistencia, Chaco.

En: http://hum.unne.edu.ar/revistas/geoweb/default.htm 
Revista Geográfica Digital. IGUNNE. Facultad de Humanidades. UNNE. Año 13. № 26. Julio - Diciembre 2016. ISSN 1668-5180 Resistencia, Chaco

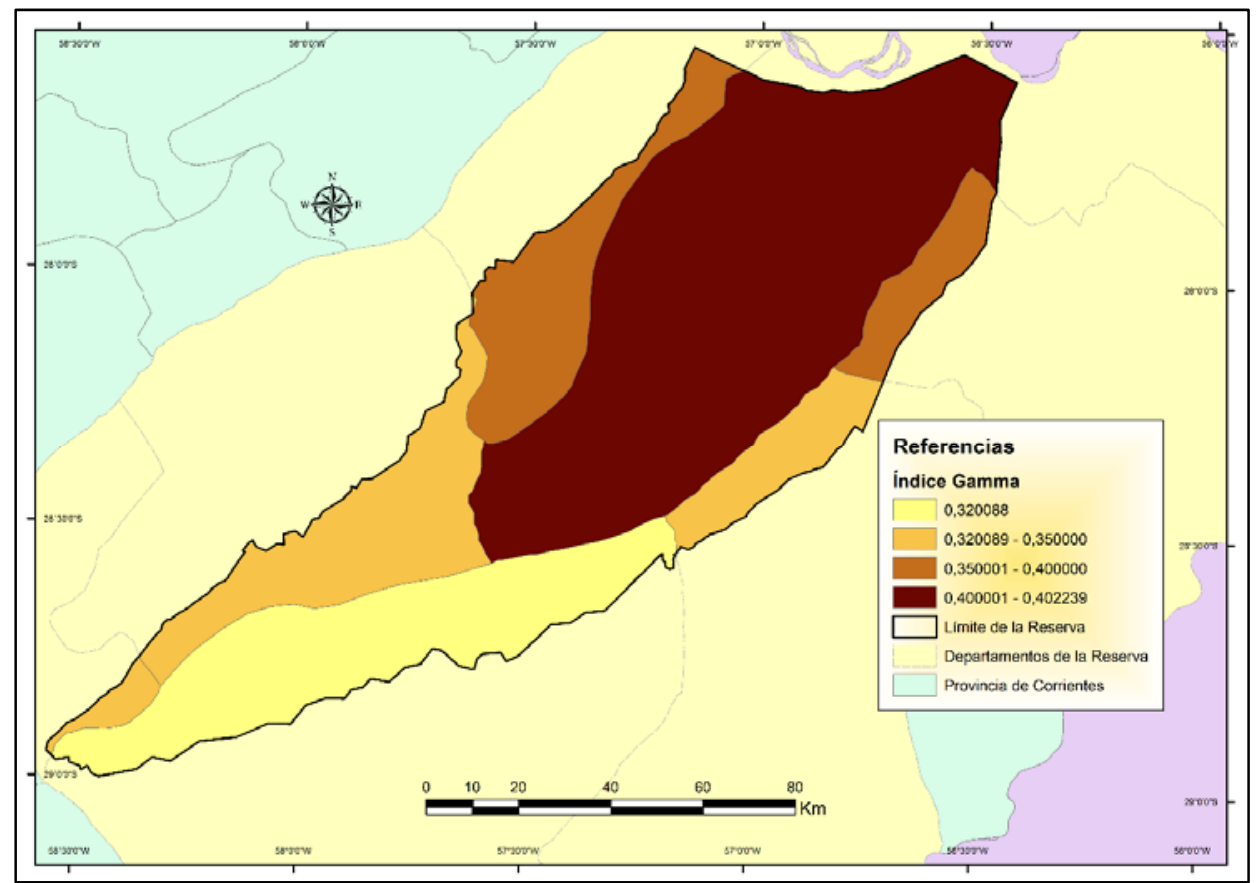

Fuente: Elaboración propia en base a los datos proporcionados por la Dirección Provincial de Vialidad de Corrientes y digitalización propia por PI $12 \mathrm{IH} 01$.

\section{CONSIDERACIONES FINALES}

El área de los Esteros del Iberá, se caracteriza por tener una red vial distribuida de manera heterogénea, con una escasa accesibilidad y conexión, producto de una densidad relativamente baja para la superficie que posee y una fuerte condicionante impuesto por las características naturales; estas son las cuestiones que surgen del análisis como limitantes a la hora de tratar de mejorar la conexión general en este territorio.

Respecto a las fuentes de información empleadas en este trabajo no se pretendió definir cuál es mejor, sino más bien complementar ambas ya que varían es sus escalas de relevamiento, lo que nos da indicios acerca de qué tipo de fuente emplear a la hora de realizar un análisis de la red vial en una espacio tan extenso como la Reserva Natural.

Desde el punto de vista metodológico, la Teoría de Grafos es un soporte metodológico sólido en el análisis de la red vial de un espacio determinado, potenciada aún más con el uso de los SIG. En este sentido, es importante enfatizar cómo dependiendo del origen de los datos analizados -IGN o PI 12IH01, que varían principalmente en la escala de relevamiento-, se presentan resultados muy distintos. Tanto en el índice Alfa como Gamma, para la red vial del IGN su valor total es superior al de la red digitalizada, a pesar de tener una menor extensión sobre el territorio.

Una línea de trabajo a explorar tiene que ver con tratar de superar el inconveniente impuesto por la delimitación de la división administrativa al momento de obtener los índices, por ejemplo utilizando una grilla reticular más reducida, ya que la superficie de los departamentos influye de manera decisiva en los cálculos. Además, para lograr una explicación más acabada de la configuración de la red vial, consideramos de vital de importancia incorporar otras variables de tipo socio-económicas y del medio

Publicado en formato digital: Prof. Federico Carlos Arias, Dr. Osvaldo Daniel Cardozo y Mgter. Miguel Alejandro Parras. ANÁLISIS DE CONECTIVIDAD Y DENSIDAD DE LA RED VIAL EN LA RESERVA NATURAL DEL IBERÁ CON SISTEMAS DE INFORMACIÓN GEOGRÁFICA (SIG). Revista Geográfica Digital. IGUNNE. Facultad de Humanidades. UNNE. Año 13. № 26. Julio - Diciembre 2016. ISSN 1668-5180 Resistencia, Chaco.

En: http://hum.unne.edu.ar/revistas/geoweb/default.htm 
natural, que se puedan combinar con los análisis de accesibilidad o centralidad y de esta forma tener una visión más integral de las infraestructuras de transporte y su entorno.

Por último, vinculado a las propuestas de intervención sobre el territorio que mejoren la conexión y movilidad, requiere no solo tener en cuenta las condiciones geográficas, sino también la situación política, las actividades económicas, criterios ambientales fijados, entre otras, ya que como resultado del conjunto de intereses privados y estatales, surgirá el modelo territorial a desarrollar en los próximos años. Sin embargo, en las propuestas de intervención deberá primar siempre un criterio más bien conservacionista antes que el desarrollista, por tratarse de una Reserva Natural.

\section{BIBLIOGRAFÍA}

Arias, F.C. y Parras, A.M. 2014. Configuración de la red vial en el área de los Esteros del lberá. XV Encuentro de Profesores en Geografía del Nordeste. Facultad de Humanidades. Universidad Nacional del Nordeste. Resistencia, Argentina.

Arias, F.C, y Cardozo, O.D. 2015. Análisis Espacial de la Red Vial en el Área de los Esteros del lberá, con Sistemas de Información Geográfica. III ${ }^{\circ}$ Jornada Provincial de Infraestructuras de Datos Espaciales y $\mathrm{V}^{\circ}$ Reunión de Usuarios de la Tecnologías de la Información Geográfica del NEA. Laboratorio de Tecnologías de la Información Geográfica. UNNE-CONICET. DIT-Ministerio de Planificación y Ambiente. Resistencia, Argentina.

Insaurralde, A.J. y Cardozo, O.D. 2010. Análisis de la Red Vial de la Provincia de Corrientes con Teoría de Grafos y Sistemas de Información Geográfica. Geográfica Digital, 13: 1-15. Recuperado de: http://hum.unne.edu.ar/revistas/geoweb/Geo13/archivos/insaur09.pdf

Izquierdo, R. 2001. Transportes. Un enfoque integral. Tomo I. Transporte y Economía del Transporte. Colegio de Ingenieros de Caminos, Canales y Puertos. Madrid, España. 609 p.

Manoiloff, R.A. y Rey, W. 2004. Atlas Geográfico de la Provincia de Corrientes. Geográfica, 12: 73 p. Instituto de Geografía. Facultad de Humanidades. UNNE. Resistencia, Argentina.

Manoiloff, R.A. y Rey, W. 2005. Atlas Geográfico de la Provincia de Corrientes. Geográfica, 13: 41 p. Instituto de Geografía. Facultad de Humanidades. UNNE. Resistencia, Argentina.

Moldes Teo, F.J. 1995. Tecnología de los Sistemas de Información. Ra-Ma. Madrid, España. 232 p.

Potrykowski, M. y Taylor, Z. 1984. Geografía del Transporte. Elena Panteleeva (trad.) Editorial Ariel. Barcelona, España. 303 p.

Seguí Pons, J.M. 1995. Análisis y estructuración de las redes en el espacio. En: A. Gamir Orueta (ed.), Prácticas de Análisis Espacial. Oikos-Tau Ediciones. Barcelona, España.

Seguí Pons, J.M. y Martínez Reynés, M.R. 2004. Geografía de los Transportes. Universitat de les Illes Balears. Palma de Mallorca, España. 444 p.

Seguí Pons, J.M. y Petrus Bey, J.M. 1991. Geografía de Redes y Sistemas de Transporte. Editorial Síntesis. Madrid, España. 231 p.

Wong, D.W.S and Lee, J. 2005. Statistical Analysis of Geographic Information. Editions John Wiley. New Jersey, Estados Unidos. 464 p.

INDEC. Instituto Nacional de Estadística y Censos. Censo Nacional de Población, Hogares y Viviendas 2010. Recuperado de: http://www.indec.gob.ar/

Publicado en formato digital: Prof. Federico Carlos Arias, Dr. Osvaldo Daniel Cardozo y Mgter. Miguel Alejandro Parras. ANÁLISIS DE CONECTIVIDAD Y DENSIDAD DE LA RED VIAL EN LA RESERVA NATURAL DEL IBERÁ CON SISTEMAS DE INFORMACIÓN GEOGRÁFICA (SIG). Revista Geográfica Digital. IGUNNE. Facultad de Humanidades. UNNE. Año 13. № 26. Julio - Diciembre 2016. ISSN 1668-5180 Resistencia, Chaco. 
Moreno Jiménez, A. 2008. Sistemas y Análisis de la Información Geográfica. Manual de autoaprendizaje con ArcGIS. Editorial Ra-Ma. Madrid, España. 940 p.

Publicado en formato digital: Prof. Federico Carlos Arias, Dr. Osvaldo Daniel Cardozo y Mgter. Miguel Alejandro Parras. ANÁLISIS DE CONECTIVIDAD Y DENSIDAD DE LA RED VIAL EN LA RESERVA NATURAL DEL IBERÁ CON SISTEMAS DE INFORMACIÓN GEOGRÁFICA (SIG). Revista Geográfica Digital. IGUNNE. Facultad de Humanidades. UNNE. Año 13. № 26. Julio - Diciembre 2016. ISSN 1668-5180 Resistencia, Chaco.

En: http://hum.unne.edu.ar/revistas/geoweb/default.htm 